\author{
Military Technical College \\ Kobry Elkobbah, \\ Cairo, Egypt \\ May 25-27,2010
}

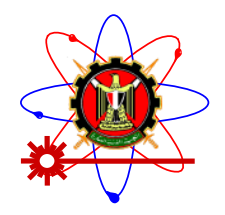

$5^{\text {th }}$ International Conference on Mathematics and Engineering Physics (ICMEP-5)

\title{
EM-14
}

\section{ON JUMP- CRITICAL ORDERED SETS}

\author{
E. M. Badr \\ Mathematics and computer Science Department, Faculty of Science, Benha University, \\ Benha, Egypt. Email: badrgraph@gmail.com \\ and
}

\begin{abstract}
M. I. Moussa
Faculty of computer \& information Benha University, Benha, Egypt,

E-mail:moussa_6060@yahoo.com
\end{abstract}

\begin{abstract}
For an ordered set $P$ and for a linear extension $L$ of $P$, Let $s(P, L)$ stand for the number of ordered pairs $(x, y)$ of elements of $P$ such that $y$ is an immediate successor of $x$ in $L$ but $y$ is not even above $x$ in $P$. Put $s(P)=\min \{s(P, L): L$ linear extension of $P\}$, the jump number of $P$. Call an ordered set $P$ is jump-critical if $\mathrm{s}(P-\{x\})<s(P)$ for any $x \in P$. We introduce some theory about the jump-critical ordered sets with jump number four. Especially, we introduce a complete list of the jump-critical ordered sets with jump number four ( it has four maximal elements). Finally, we prove that a $k$-critical ordered set is a $k$-tower ( its width is $2, k>1$ ).

KEYWORDS: Jump number, jump-critical ordered sets.
\end{abstract}




\section{INTRODUCTION}

Let $P$ be a poset and $L$ be a linear extension of $P$. Every linear extension $L$ of a finite ordered set $P$ can be expressed as the linear sum $C_{1} \oplus C_{2} \oplus \ldots \oplus C_{\mathrm{m}}$ of chains $C_{\mathrm{i}}$ of $P$ so labeled that $\sup _{P} C_{\mathrm{i}}$ 织 $\inf _{P} C_{\mathrm{i}+1}$ in $P$.

( The linear sum $A \oplus B$ of ordered sets $A$ and $B$ is the set $\mathrm{A} \cup \mathrm{B}$ ordered so that $a \leqq b$ provided that $a \in A$ and $b \in B$, or else, $a \leqq b$ in $A$ or, $a \leqq b$ in $B$ ).

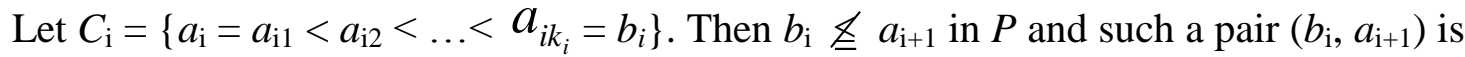
called a jump (or set up) of the linear extension $L$, which is said to have $m$ - 1 jumps. We write $s(P, L)=m-1$. Note that $\mathrm{a}_{\mathrm{i}+1}$ covers $\mathrm{b}_{\mathrm{i}}$ in $L$, although $\mathrm{a}_{\mathrm{i}+1} \not \mathrm{b}_{\mathrm{i}}$ in $P$ itself. We put $s(P)=\min \{s$ $(P, L) \mid L$ linear extension of $P$ \}. This problem finds its practical settings too. Let the elements of $P$ represent certain jobs to be performed one at a time by a single processor while the order of $P$ imposes precedence constraints upon these jobs. Then an optimal linear extension of $P$ is just a schedule of the jobs which minimizes the number of " set up" between unrelated jobs.

Observe that $s(P) \geq s(P-\{x\}) \geq s(P)-1$ for any $x \in P$. A poset $P$ is called jumpcritical if $s(P-x)<s(P)$, for every element $x \in P$. If $P$ is jump-critical with $s(P)=m$, then we say that $P$ is $m$-jump-critical. It is easy to see that every ordered set $P$ contains a jump-critical subset $K$ with $s(P)=s(K)$. It may be that jump-critical ordered sets tell us much about the problem determining $s(P)$ - even about constructing "optimal" linear extensions for $P$, that is, ones for which $s(P, L)=s(P)$. The ordered set illustrated in Fig. 1 is jump-critical. Obviously, $\left.s\left(P-\left\{a_{41}\right\}\right)<s P\right)$. But to verify that $s\left(P-\left\{a_{31}\right\}\right)<4$, for instance, requires a different chain decomposition : $P-\left\{a_{31}\right\}=C_{2} \oplus C_{4} \oplus C_{5} \oplus\left\{a_{11}<a_{12}<a_{32}\right\}$. It is a good exercise to check all eight cases.

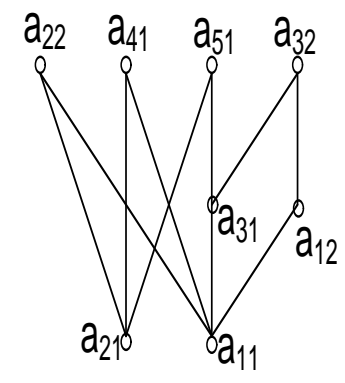

$s(P)=s(P, L)=4$

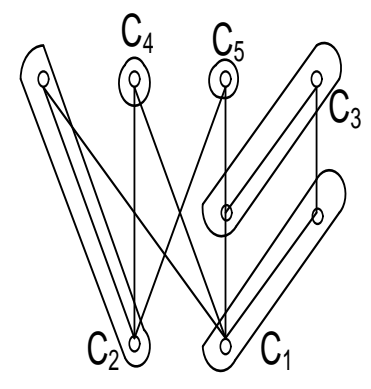

$P=\bigcup_{i=1}^{5} C_{i}$

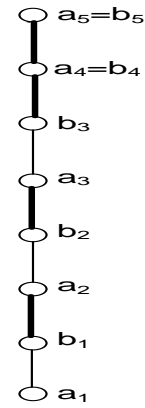

$$
L=\bigoplus_{i=1}^{5} C_{i}
$$

\section{Figure 1}

The purpose of this paper is to stimulate activity on the jump number of an ordered set by recording several important examples. In section 2, we introduce some special methods to construct jump-critical ordered sets. In section 3 , we introduce the complete lists of 1-jumpcritical, 2-jump-critical, 3-jump-critical ordered sets and some theorems about 4-jump-critical ordered sets.

\section{Special Methods to Construct Jump-Critical Ordered Sets.}


In this section we present special methods for constructing jump-critical posets. An $n$ element antichain is jump-critical. In fact, it is fairly obvious that the disjoint sum of jumpcritical ordered sets is jump-critical. In addition, $s\left(P_{1}+P_{2}\right)=s\left(P_{1}\right)+s\left(P_{2}\right)+1$. It is equally obvious that the linear sum of jump-critical ordered sets is jump-critical. Also $s\left(P_{1} \oplus P_{2}\right)=s$ $\left(P_{1}\right)+s\left(P_{2}\right)$. These are special cases of a more general construction. Let $\mathrm{P}$ be an ordered set and each $x \in P$, let $P_{x}$ be an ordered set. The lexicographic sum $\sum_{x \in P} P_{x}$ is the set $\bigcup_{x \in P} P_{x}$ ordered so that $u \leqq v$ if, for some $x \in P, u \in P_{x}, v \in P_{x}$ and $u \leqq v$ in $P_{x}$, or else, $u \in P_{x}, v \in P_{y}$, for some $x<y$ in $P$. It is implicit in M. Habib [5] that the lexicographic sum $\sum_{x \in P} P_{x}$ of critical ordered sets $P_{x}$ is itself critical, as long as each $\left|P_{x}\right|>2$. M. H. El-Zahar and I. Rival introduced a new method which gets jump-critical ordered sets by the theorem 1 [2].

Theorem 1. Let $P_{1}$ and $P_{2}$ be finite jump-critical ordered sets. Any ordered set obtained from $P_{1}$ and $P_{2}$ by gluing a maximal element of $P_{1}$ with a maximal element of $P_{2}$ is jump-critical and, in this case, the jump number is $s\left(P_{1}\right)+s\left(P_{2}\right)$. If $\left|\max P_{1}\right|=\left|\max P_{2}\right|=2$ then the ordered set obtained from $P_{1}$ and $P_{2}$ by gluing max $P_{1}$ with max $P_{2}$ is jump-critical and, in this case, the jump number is $s\left(P_{1}\right)+s\left(P_{2}\right)-1$.

This gluing construction can be used to construct an example of jump-critical ordered set in which an "optimal" linear extension uses a long chain (see Fig. 2).

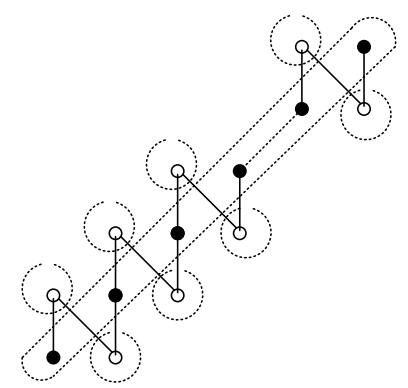

\section{Figure 2}

There is an obvious question that arises fro the second part of Theorem 1: does the gluing the construction produce a jump-critical ordered set if there are more than two maximal elements? . This question is open until now.

\section{3. (1-2-3-4)-jump-critical ordered sets}

In this section, we introduce the complete lists of 1-jump-critical, 2-jump-critical, 3jump-critical ordered sets and some theorems about 4-jump-critical ordered sets.

Obviously, the only jump-critical ordered sets $P$ with $s(P)=0$ is the singleton. If $s(P$ )$=1$ then, of course, $P$ must contain a noncomparable pair of elements. So, if $P$ is jumpcritical then $P$ must be a two-element antichain. Suppose $P$ is jump-critical and $s(P)=2$. $P$ may be a three-element antichain. The only other possibility is that $P$ is the " four-cycle". Thus, either $P \cong 1+1+1$ or $P \cong(1+1) \oplus(1+1)$. 
The only jump-critical ordered set with jump number one, the twoelement antichain.
The two jump-critical ordered Sets with jump number two, the threeelement antichain, and the four-cycle.

\section{Figure 3}

M. H. El-Zahar and I. Rival [2] introduced the complete list of the jump-critical ordered sets with jump number three which has fourteen jump-critical ordered sets. These are, up duality, the ordered sets illustrated in Figure 4.
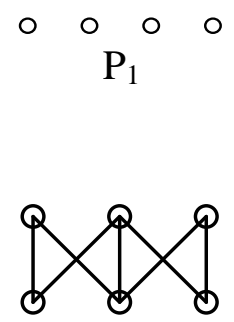

$\mathrm{P}_{5}$

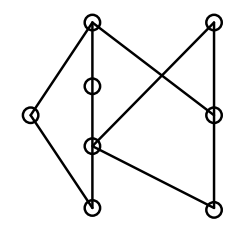

$\mathrm{P}_{9}$
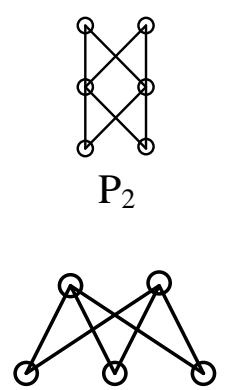

$\mathrm{P}_{6}$

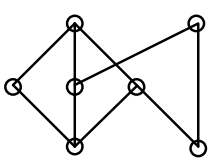

$\mathrm{P}_{10}$

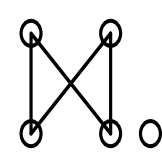

$\mathrm{P}_{3}$

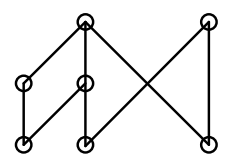

$\mathrm{P}_{7}$

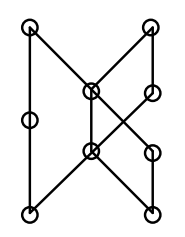

$\mathrm{P}_{11}$

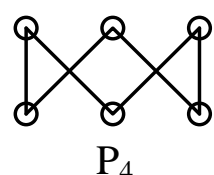

$\mathrm{P}_{4}$

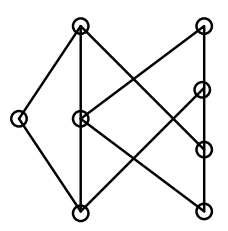

$\mathrm{P}_{8}$

Figure 4

Let $P$ be a finite ordered set. For an element $a$ in $P$ put $D(a)=\{x \in P \mid x \leqq a\}$, a down set in $P, U(a)=\{x \in P \mid x \geqq a\}$, an upper set in $P$. Following M. H. El-Zahar and J. H. Schmerl [3] call the element a accessible in $P$ if $D(a)$ is a chain in $P$. For instance, each minimal element of $\mathrm{P}$ is accessible. Let $w(P)$ stand for the width of $P$, the size of a maximum-sized antichain. According to Dilworth's chain decomposition theorem [1], $P$ is the (disjoint) union of $w(P)$ chains. For maximum-sized antichains $A, B$ in $P$ we write $A \leqq B$ whenever for $a \in A$ there is $b \in B$ satisfying $a \leqq b$. ( It follows, in this case that, for each $b \in B$ there is $a \in A$ satisfying $a \leqq b$, too). In this way the set of maximum-sized antichains of $P$ is ordered: there is greatest (highest) antichain and a least (lowest) antichain. As matter of fact, the set of maximum-sized antichains is a distributive lattice in which $A \vee B=\max (A \cup B)$ and $A \wedge B=\min (A \cup B)$ (R. P. Dilworth[1]). A tower of height $k$ ( or $k$-tower) is a linear sum of $k$-comparable elements [4]. Obviously, a $k$-tower is $k$-critical with width two.

Theorem 2. Let $P$ be a $k$-jump-critical ordered set with width 2 where $k>1$. Then $P$ is a $k$ tower. 
Proof. We use induction on $k$. For $k=2$, the only poset which satisfies the criteria of the theorem is the 4-alternating-cycle $2 \oplus 2$. Thus, the result is true for $k=2$, and assume that it is true for jump-critical posets with jump-number less than $k$. Now we want to prove that it is true for jump-critical posets with jump-number $k$.

since $w(P)=2$ then it is the union of two chains $C_{1}$ and $C_{2}$. Put $x_{\mathrm{i}}=\inf _{\mathrm{p}} C_{i}$ for $i=1,2$.

As $P$ is jump - critical then $x_{1} \neq x_{2}$. Let $a_{\mathrm{i}}$ be the maximal accessible element on $C_{i} ; i=$ 1,2. See Fig. 5.

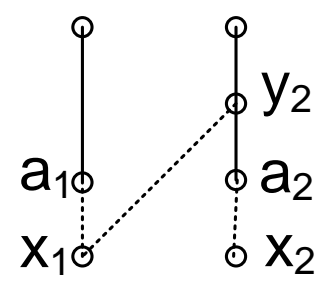

\section{Figure 5}

We want to prove that $a_{\mathrm{i}}=x_{\mathrm{i}}, i=1,2$. Suppose not, say $a_{1}>x_{1}$. Put $P^{`}=P-\left\{a_{1}\right\}$. As $P$ is jump-critical then $s\left(P^{\prime}\right)=k-1$. Let $L$ be a linear extension of $P^{`}$ with $k-1$ jumps, say $L=C_{1}$ ' $\oplus \ldots \oplus C^{n}{ }_{k}$ where each $C^{n}$ is a chain, $i=1,2, \ldots, k$. If $x_{1} \in C^{n_{1}}$ then $C^{n} \cup\left\{a_{1}\right\}$ is also a chain. So, we can replace $C_{1}$ on $L$ by $C{ }_{1} \cup\left\{a_{1}\right\}$ which gives a linear extension of $P$ with only $k$ - 1 jumps. This a contradiction. So, $x_{1} \notin C_{1}$ which implies that $C_{1}=x_{2} \ldots a_{2}$. Now $x_{1}$ $\in C_{2}$. If $C_{2} \cap C_{2}=\varnothing$ then $\mathrm{C}_{2}^{\prime} \cup\left\{a_{1}\right\}$ is a chain. Again, we can replace $\mathrm{C}_{2}^{\prime}$ by $\mathrm{C}_{2}^{\prime} \cup\left\{a_{1}\right\}$ to get $s(P)=k-1$; a contradiction. Therefore $C_{2}$ has the form $C_{2}=x_{1} \ldots y_{2} \ldots m$ where $y_{2}$ is the element that covers $a_{2}$ on $C_{2}$ and $m=\max C_{2}$ is some element in $C_{2}$ (possibly $m=y_{2}$ ). Now we can replace $C_{1}$ and $C_{2}^{\prime}$ respectively by $C^{{ }^{\prime}}{ }_{1}$ and $C_{{ }_{2}}^{\prime}$ where $C^{{ }_{1}}{ }_{1}=x_{1} \ldots a_{1}$ and $C^{\prime \prime}{ }_{2}$

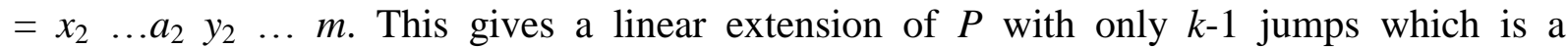
contradiction. We conclude that $a_{1}=x_{1}$ and similarly $a_{2}=x_{2}$. Now $P-\left\{x_{1}, x_{2}\right\}$ has jump number $k-1$ and, by induction, contains a $(k-1)$ tower. This $(k-1)$ tower together with $\left\{a_{1}, a_{2}\right\}$ forms a $k$-tower. This must be all of $P$. This completes the proof of the Theorem.

\section{Theorem 3}

There are precisely forty for jump-critical ordered sets ( without isolated element) with four maximal elements and $s(P)=4$. These are, up duality, the ordered sets illustrated in Fig. (6).

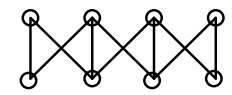

A

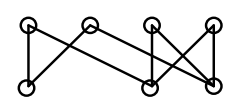

$\mathrm{E}$
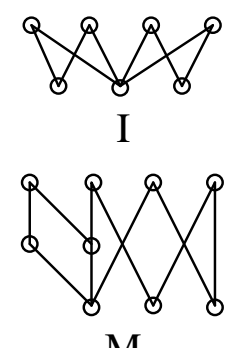

$\mathrm{M}$

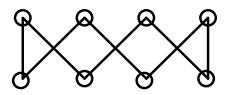

B

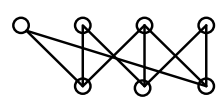

$\mathrm{F}$
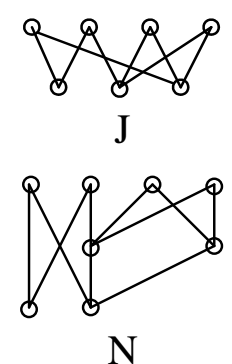

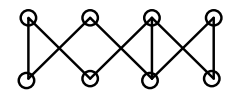

$\mathrm{C}$

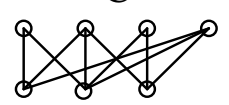

$\mathrm{G}$

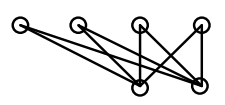

K

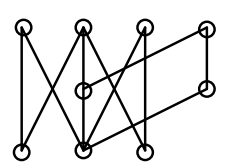

$\mathrm{O}$

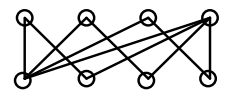

D

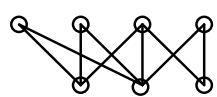

$\mathrm{H}$

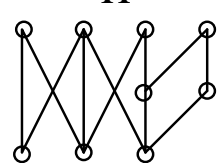

$\mathrm{L}$

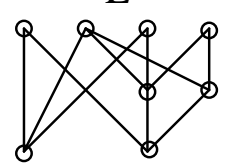

$\mathrm{P}$ 


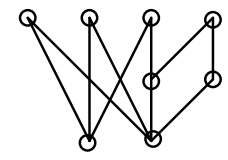

$\mathrm{Q}$

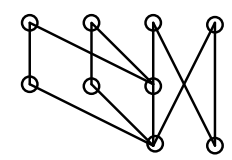

$\mathrm{U}$

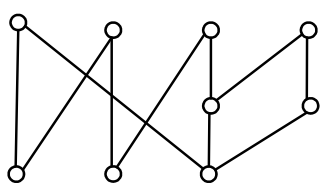

$\mathrm{V}$

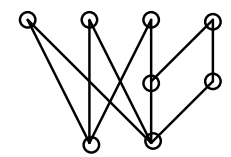

$\mathrm{S}$

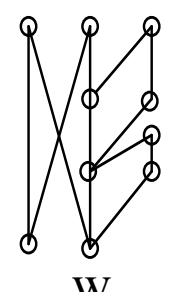

$\mathrm{W}$

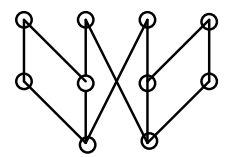

$\mathrm{T}$

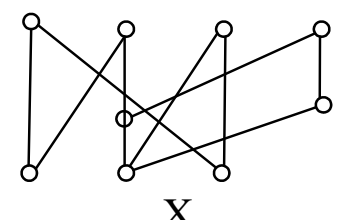

$\mathrm{X}$

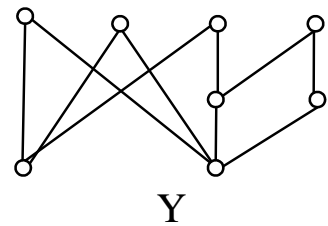

Figure 6

Proof of Theorem 3. It is straightforward, if somewhat laborious, to verify that each of the ordered sets illustrated in Fig. 6 has jump number four, four maximal elements and that each is jump critical without isolated element.

Let $P$ be 4-jump-critical and has four maximal elements (without isolated element). For contradiction, suppose that $P$ contains no subset isomorphic to any of the posts illustrated in figure 6 .

Since $P$ is 4-jump-critical with $w(P)=4$, then $P=C_{1} \cup C_{2} \cup C_{3} \cup C_{4}$ (disjoint chains). Put $a_{i}=\inf _{P} C_{i}$ and $b_{i}=\sup _{P} C_{\mathrm{i}}$ for $\mathrm{i}=1,2,3,4$. Let us suppose that both $\left\{a_{1}, a_{2}\right.$, $\left.a_{3}, a_{4}\right\}$ is an antichain and $\left\{b_{1}, b_{2}, b_{3}, b_{4}\right\}$ is maximal elements antichain. If $b_{i}$ 's is accessible, then $a_{i} \neq b_{i},\left|D\left[b_{i}\right] \cap\left\{a_{1}, a_{2}, a_{3}, a_{4}\right\}\right| \geq 2$ and, dually, $\left|D\left[a_{i}\right] \cap\left\{b_{1}, b_{2}, b_{3}, b_{4}\right\}\right| \geq 2$. If follows that $\left\{a_{1}, a_{2}, a_{3}, a_{4}, b_{1}, b_{2}, b_{3}, b_{4}\right\}$ is isomorphic to $A$ or $B$ or $C$ or $D$. Or that $\left\{a_{1}, a_{2}, a_{3}\right.$, $\left.b_{1}, b_{2}, b_{3}, b_{4}\right\}$ contains $E\left(E^{d}\right)$ or $F\left(F^{d}\right)$ or $G\left(G^{d}\right)$ or $H\left(H^{d}\right)$ or $J\left(J^{d}\right)$.

Next, we handle the case $\left\{a_{1}, a_{2}, a_{3}, a_{4}\right\}$ is not antichain. Let $\left\{\mathrm{c}_{1}, c_{2}, c_{3}, c_{4}\right\}$ be infimum of all four-element antichain in $P$.

One of $c_{i}$ 's must be less than one of $b_{i}$ 's, only, say $c_{1}<b_{1}$, for otherwise the proper subset $\left(\bigcup_{i=1}^{4} U\left[c_{i}\right]\right)$ of $P$ has jump number four. If $\left(P-U\left[c_{1}\right]\right)$ contains four-element antichain, $\left\{x_{1}, x_{2}, x_{3}, x_{4}\right\}$ then $c_{1}$ must be comparable to one of these $x_{\mathrm{i}}$ 's (say) $x_{1}$. But $x_{1}>c_{1}$, since $x_{1} \notin U\left[c_{1}\right]$ and if

$x_{1}<c_{1}$ then $\left\{c_{1}, c_{2}, c_{3}, c_{4}\right\}$ is not the lowest four-element antichain in $P$. Therefore, $w\left(P-U\left[c_{1}\right]\right)=3$ and we can assume that, $\left(P-U\left[c_{1}\right]\right)=C_{2} \cup C_{3} \cup C_{4}$ so that $U\left[c_{1}\right]=C_{1}$. Let $\left\{d_{2}, d_{3}, d_{4}\right\}$ and $\left\{b_{2}, b_{3}, b_{4}\right\}$ be respectively, the lowest and highest, three-element antichain in $C_{2} \cup C_{3} \cup C_{4}$ where, say, $d_{i}, b_{i} \in C_{i}$ for both $i=2,3,4$ since $s\left(C_{2} \cup C_{3} \cup C_{4}=3\right)$ then $\left\{d_{2}, d_{3}\right.$, $\left.d_{4}, b_{2}, b_{3}, b_{4}\right\}$ is isomorphic to the following posets

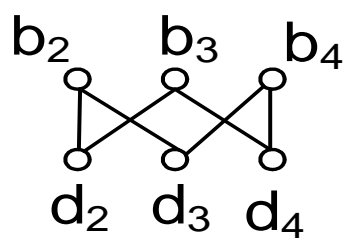

or

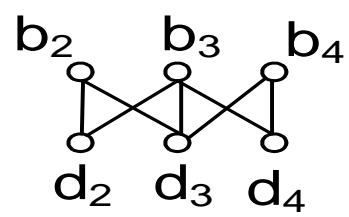

$\begin{array}{lll}\mathrm{d}_{2} & \mathrm{~d}_{3} & \mathrm{~d}_{4}\end{array}$ 
Neither $b_{\mathrm{i}}$ is above $c_{1}$. Also $c_{1}$ can not below $d_{i}$ 's, otherwise $c_{1}<$ one of $b_{i}$ 's only. Moreover $c_{1}$ $>d_{2}$ or $c_{1}>d_{3}$ or $c_{1}>d_{4}$. Otherwise $c_{1}$ is an isolated element in $P$. Therefore $\min (P)=\min$ $\left(C_{2} \cup C_{3} \cup C_{4}\right)$. For otherwise $P$ would have a unique minimal element.

If $c_{1}>d_{2}, c_{1}>d_{3}$ and $c_{1}>d_{4}$ then

$\left\{\mathrm{c}_{1}, d_{2}, d_{3}, d_{4}, b_{2}, b_{3}, b_{4}\right\} \cong E$ or $\left\{\mathrm{c}_{1}, d_{2}, d_{3}, d_{4}, b_{2}, b_{3}, b_{4}\right\} \cong G$

If $c_{1}>$ the two elements of $\left\{d_{2}, d_{3}, d_{4}\right\}$ then

$$
\left\{c_{1}, d_{2}, d_{3}, d_{4}, b_{2}, b_{3}, b_{4}\right\} \cong E \text { or }\left\{c_{1}, d_{2}, d_{3}, d_{4}, b_{2}, b_{3}, b_{4}\right\} \cong H .
$$

We may then suppose that $c_{1}>d_{2}, c_{1}>d_{3}$ and $c_{1}>d_{4}$. Since $b_{1}=\sup _{P} C_{1}$, let us suppose that $b_{1}>b_{2}, b_{1}>b_{3}$ and $b_{1}>b_{4}$ then there exists an element $d \in C_{2} \cup C_{3} \cup C_{4}$ such that $d \neq d_{2}, d<$ $b_{1}$ and $c_{1} \| d$.

Otherwise, $c_{1}$ is an accessible in the $P^{d}$, as $\left(P-U\left[c_{1}\right]\right)$ has width three and jump number three so it must contain

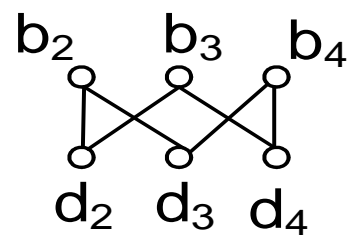

or

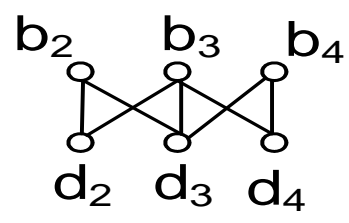

So that any of these figures, with $c_{i}$ is a subposet of $P$ contains isolated element $c_{1}$. If $b_{1}>$ one of $\left\{d_{3}, d_{4}\right\}$ then $\left\{b_{1}, b_{2}, b_{3}, b_{4}, d_{2}, d_{3}, d_{4}\right\} \cong F$ or $\cong H$. Otherwise

(i) $\quad d_{2}<d<b_{2}, d \| b_{3}$ and $d \| b_{4}$ or

(ii) $\quad d_{3}<d<b_{5}, d\left\|b_{2}, d\right\| b_{4}$ and $d \| b_{1}$

If (i) satisfies then $\left\{b_{1}, \mathrm{c}_{1}, d, b_{2}, b_{3}, b_{4}, d_{2}, d_{3}, d_{4}\right\}$ isomorphic to $L, M$, V or X; if (ii) satisfies then $\left\{b_{1}, c_{1}, b_{2}, b_{3}, b_{4}, d_{2}, d_{3}, d_{4}, d\right\}$ isomorphic to $U$. Now let $\left\{f_{2}, f_{3}\right\}$ and $\left\{b_{2}, b_{3}, b_{4}\right\}$ be, respectively, the lowest and height, two-element antichain and three-element antichain in $C_{2}$ $\cup C_{3} \cup C_{4}$ where $f_{i}, b_{i} \in C_{\mathrm{i}}$ for $i=2,3,4$. Since $s\left(C_{2} \cup C_{3} \cup C_{4}\right)=3$ then $P$ contains

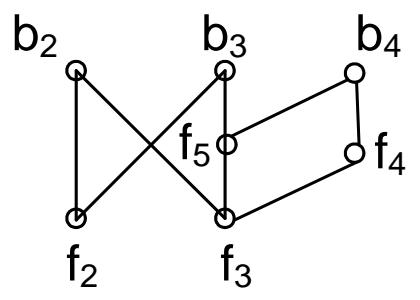

or

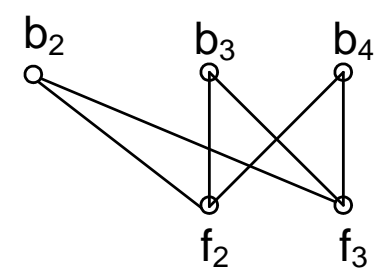

Neither $b_{i}$ is above $c_{i}$. Also $c_{i}$ can not below $f_{i}$ 's otherwise, $c_{1}<$ one of $b_{i}$ 's only. Moreover $c_{1}$ $>f_{2}$ or $c_{1}>f_{3}$ or $c_{1}>f_{4}$ or $c_{1}>f_{5}$ otherwise $c_{1}$ is isolated element in $P$. Therefore $\min (P)=$ $\min \left(C_{2} \cup C_{3} \cup C_{4}\right)$, for otherwise $P$ would have a unique minimal element.

If $c_{1}>f_{2}$ and $c_{1}>f_{3}$, then $\left\{c_{1}, b_{2}, b_{3}, b_{4}, f_{2}, f_{3}\right\} \cong K$.

If $c_{1}>f_{2}$ and $c_{1}>f_{4}$ and $c_{1}>f_{5}$; since $c_{1}\left\|b_{2}, c_{1}\right\| b_{3}$ and $c_{1} \| b_{4}$ then $\left\{c_{1}, b_{2}, b_{3}, b_{4}, f_{2}, f_{3}, f_{4}\right.$, $\left.f_{5}\right\} \cong P$. If $c_{1}>f_{2}, c_{1}>f_{4}$ and $c_{1} \| f_{5}$ since $c_{1}\left\|b_{2}, c_{1}\right\| b_{3}$ and $c_{1} \| b_{4}$ then $\left\{c_{1}, b_{2}, b_{3}, b_{4}, f_{2}\right.$, $\left.f_{3}, f_{4}, f_{5}\right\} \cong R, \mathrm{O}$ or $\mathrm{Y}$.

Now, if $c_{1}>f_{2}$ and $c_{1}>f_{3}$; since $b_{1}=\sup _{P} C_{1}$, let us suppose that $b_{1}>b_{2}, b_{1}>b_{3}$ and $b_{1}>b_{4}$ then there is an element $\mathrm{f} \in C_{2} \cup C_{3} \cup C_{4}$ such that $f \neq f_{2}$;

$f<b_{1}$ and $c_{l}, f$ are incomparable, otherwise $c_{1}$ is an accessible in the $P^{d}$. As $\left(P-U\left[c_{1}\right]\right)$ has width three and jump number three, it must contains 

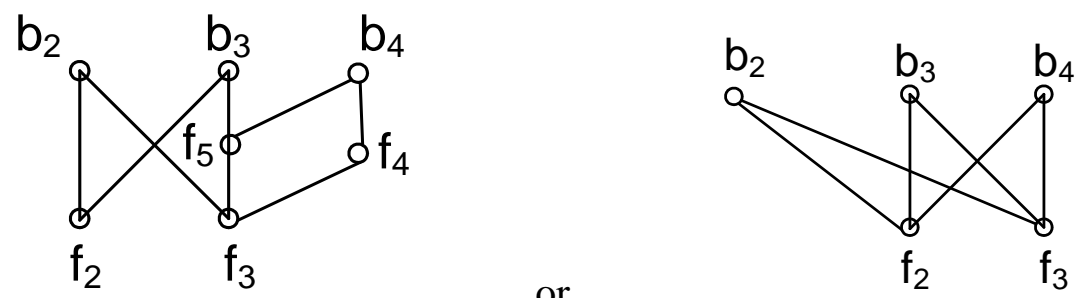

So that any of these figures with $c_{1}$ is a subposet of $P$ contains isolated element $c_{1}$. If $b_{1}>f_{3}$ then $\left\{b_{1}, b_{2}, b_{3}, b_{4}, f_{2}, f_{3}\right\} \cong K$ and $\left\{b_{1}, b_{2}, b_{3}, b_{4}, f_{2}, f_{3}, f_{4}, f_{5}\right\} \cong S$ otherwise $f_{2}<f<b_{2}$ and $f$ $\| b_{3}$ and $f \| b_{4}$ then $\left\{c_{1}, f, b_{1}, b_{2}, b_{3}, b_{4}, f_{2}, f_{3}, f_{4}, f_{5}\right\} \cong T$. If $f_{3} \prec c_{1}$ or $f_{4} \prec c_{1}$ or $f_{5} \prec c_{1}$ and $c_{1}$ $>f_{2} ;$ since $f_{5} \neq f_{3}$,

$f_{5}>f_{3}$ therefore $f_{5} \| c_{1}$, otherwise $P-\left(\bigcup_{i=1}^{4} U\left[c_{i}\right]\right)$ has jump number four. Then, if $b_{1}>f_{5}$ then $\left\{b_{1}, b_{2}, b_{3}, b_{4}, c_{1}, f_{2}, f_{3}, f_{4}, f_{5}\right\} \cong W$, if $f_{4} \prec c_{1}, b>f$ and $f>f_{4}$ then $\left\{b_{1}, b_{2}, b_{3}, b_{4}, f_{2}, f_{3}, f_{4}, f_{5}\right.$, $\left.f, c_{1}\right\} \cong V$, if $f_{5} \prec c_{1}, c_{1}>f_{4}$ and $c_{1}>f_{2}$ then $\left\{c_{1}, f_{2}, f_{3}, f_{4}, f_{5}, b_{2}, b_{3}, b_{4}\right\} \cong N$ and if $f_{5} \prec c_{1}, f_{5}$ $\prec f, b_{1}>f$ then $\left\{b_{1}, b_{2}, b_{3}, b_{4}, f_{2}, f_{3}, f_{4}, f_{5}, f, c_{1}\right\} \cong W$. Hence this theorem is proved.

\section{4- Conclusion}

In this paper, we introduced some theorems about 4-jump-critical ordered sets. Especially, we introduced a complete list of the jump-critical ordered sets with jump number four ( it has four maximal elements). Finally, we proved that a $k$-critical ordered set is a $k$ tower ( its width is $2, k>1$ ). In future, we can investigate the structure of m-jump-critical ordered sets to study the jump-number problem.

\section{References}

[1] R. P. Dilworth (1950), A decomposition theorem for partially ordered sets, Ann. Math., 51, 161-166.

[2] M. H. El-Zahar and I. Rival (1985), Examples of jump - critical ordered sets, SIAM J. Algebraic Discrete Methods. 6 (4), 713-720.

[3] M. H. El-Zahar and Schemer J.H. (1984), On the size of jump-critical ordered sets, order 1, 3-5.

[4] Hell P, Li W. and Schmerl J. H. (1986), Jump number and width, order 5 , 227-234

[5] M. Habib (1984), comparability invariants, Annals Discrete Math, 23, 331-386.

[6] M. H. El-Zahar (2000), On Jump-Critical Posets with Jump-Number Equal to Width, order 17, 93 - 101. 